\title{
VLA HI Imaging of the Low Surface Brightness Dwarf Galaxy DDO 47
}

\author{
F. Walter \\ Radioastronomisches Institut, Bonn, Germany \\ E. Brinks \\ Departamento de Astronomía, Guanajuato, México
}

\begin{abstract}
We present high resolution VLA-observations of the nearby Low Surface Brightness Dwarf Galaxy DDO 47. This object shows many hole-like structures in its neutral interstellar medium. The majority of the detected $\mathrm{HI}$-shells are found to be expanding. Their origin is therefore believed to be due to stellar winds of the most massive stars and their subsequent supernova (SN type II) explosions within regions of recent star formation (SF). Current SF in DDO 47 is predominantly present on the rims of the $\mathrm{H} \mathrm{I}$-shells suggesting propagating SF. At a projected distance of $20 \mathrm{kpc}$ (adopting a distance to DDO $47 \mathrm{of} 4 \mathrm{Mpc}$ ), a companion galaxy was detected at almost the same systemic velocity (DDO $47 \mathrm{~B}$ ). A search for an optical identification suggests that CGCG 087-033 is the optical counterpart of the companion. A preliminary dynamical analysis based on DDO 47's rotation curve yields that it is dark matter dominated (about $80 \%$ of its dynamical mass is in some non-visible form). A simple mass model suggests that DDO 47 is one of the 'thickest' dwarf galaxies studied so far.
\end{abstract}

\section{Introduction}

Recent H I-studies of nearby dwarf galaxies show that holes and shell-like structures dominate the appearance of their interstellar medium (ISM). These holes are thought to be created as a result of the formation and rapid evolution of massive stars within a group or association, via the interaction of their collective winds and supernova explosions with the surrounding ISM (see Tenorio-Tagle and Bodenheimer 1988). This picture is not without its critics (see Rhode et al. 1997; also this volume). But whatever energetic events cause these structures, they leave a much more dramatic impression on a dwarf galaxy than on a more massive spiral galaxy (Walter \& Brinks 1998). This is attributed to dwarf galaxies having a lower overall gravitational potential.

Despite the fact that $\mathbf{d w a r f}$ galaxies are ideal laboratories for ISM studies, only few papers dealing with detailed $\mathrm{H}$ I observations have been published thusfar. Examples are the Large and the Small Magellanic Clouds (see Kim et al. 1997) and Holmberg II (Puche et al. 1992). We therefore started a programme to observe a sample of nearby dwarf galaxies. The first paper, on IC 2574 has been 
submitted (Walter \& Brinks 1998). First results on a second object, DDO 47, are presented here. DDO 47 (UGC 3974) was observed in the $21-\mathrm{cm}$ line of neutral hydrogen with the NRAO Very Large Array (VLA) in its D, DnC, C and $\mathrm{B}$-configurations. In total, 16 hours were spent on source. The velocity and spatial resolution are $2.5 \mathrm{~km} \mathrm{~s}^{-1}$ and $7^{\prime \prime}$ (resulting in a linear resolution of $120 \mathrm{pc}$ at an adopted distance of $4 \mathrm{Mpc}$ ). In the course of the data reduction we discovered a companion galaxy at nearly the same systemic velocity (see Fig 1).

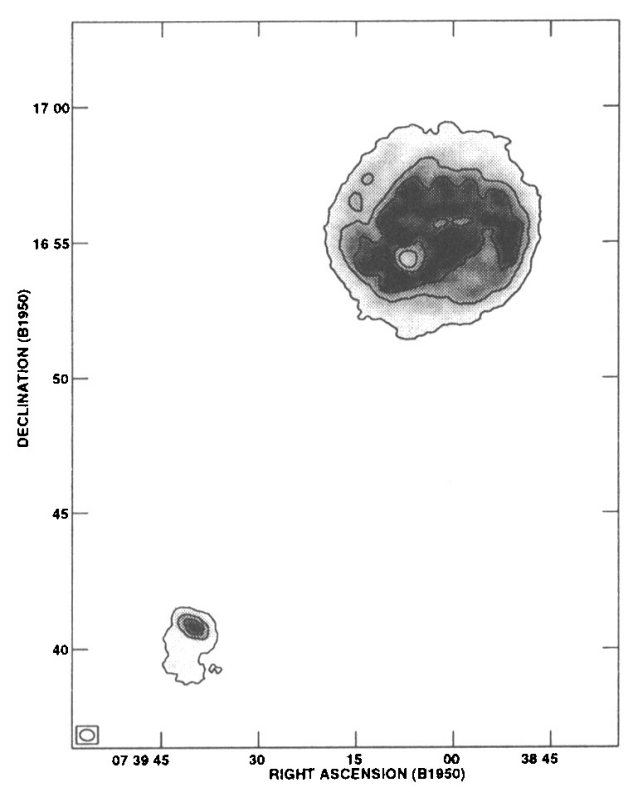

Figure 1. H I surface brightness map of DDO 47 (north) and its companion (south). The projected distance is about $20 \mathrm{kpc}$.

\section{The H I-H $\alpha$ connection of DDO 47}

In total, we detected $19 \mathrm{H} \mathrm{I-holes} \mathrm{in} \mathrm{DDO} \mathrm{47.} \mathrm{An} \mathrm{overlay} \mathrm{of} \mathrm{the} \mathrm{positions} \mathrm{and}$ sizes of the holes with the H I surface brightness map is given in Fig. 2 (left). Most of the holes were found to be expanding. This lends support to the picture that they are wind- and/or supernova driven. The theory of propagating star formation predicts that secondary sites of star formation form close to the rims of expanding $\mathrm{H}$ I holes. The idea behind this is that the holes, while expanding, sweep up ambient matter, to such a point that the mass density on the rim gets high enough for star formation to commence. To test this scenario in the case of DDO 47, the $\mathrm{H} \alpha$-regions detected by Strobel et al. (1991) were overlaid on the H I surface density map (Fig. 2, right). Note that virtually all $\mathrm{H} \alpha$ emission is situated outside the holes which at least qualitatively fits this description. 

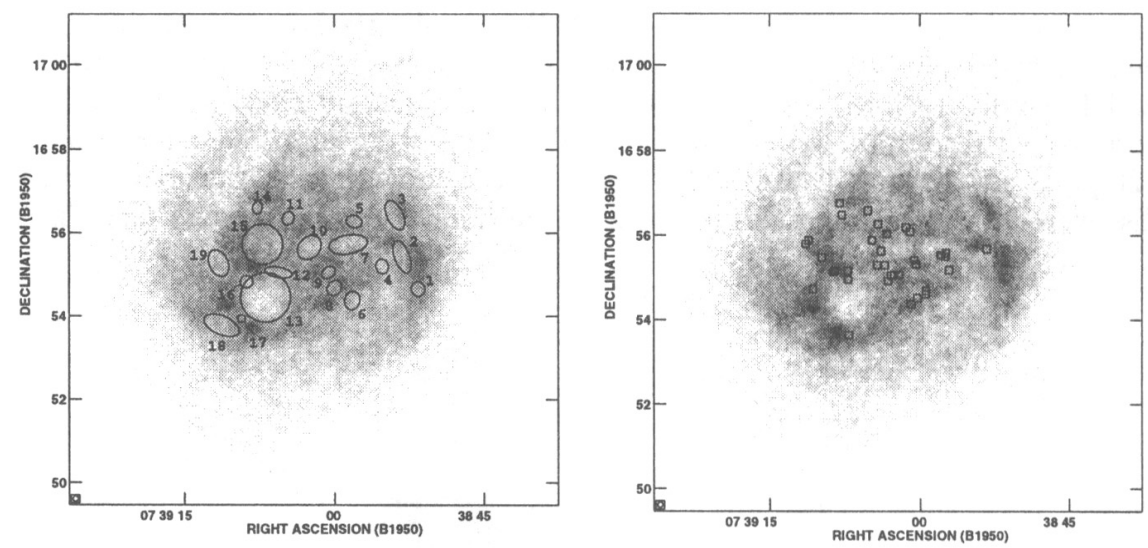

Figure 2. The distribution of the neutral hydrogen in DDO 47 overlaid with the position and sizes of the $\mathrm{HI}$ holes detected by us (left) and the H II regions from Strobel et al. (right).

\section{Dynamics of DDO 47 and the thickness of its $H$ I disk}

After a preliminary analysis of the observed rotational velocities and rotation curve fitting (see Fig. 3), the dynamical mass of DDO 47 was determined to be about $5 \times 10^{9} \mathrm{M}_{\odot}$. Assuming a gaseous mass of $3 \times 10^{8} \mathrm{M}_{\odot}$ (H I, corrected for the contribution of primordial helium) and a stellar mass of $2 \times 10^{8} \mathrm{M}_{\odot}$ (assuming a solar mass to light ratio for the stars), yields that most of the mass of DDO 47 is present in the form of Dark Matter. Fig. 3 shows the rotation curve of DDO 47 (boxes, with appropriate error bars). The triangles present the contribution from neutral hydrogen, the open square shows the contribution from the stellar population out to a radius of $4^{\prime}$. From this simple analysis it is already clear that most of the mass must be present in the form of Dark Matter. Using a simple model which relates the velocity dispersion of the gas to its scale height, we derive the thickness of the H I-layer of DDO 47 to be about $700 \mathrm{pc}$. DDO 47 is therefore one of the thickest dwarf galaxies studied so far.

\section{Summary}

- DDO 47 is a gas-rich dwarf irregular galaxy at distance of $4 \mathrm{Mpc}$.

- A companion has been detected at almost the same systemic velocity. Its H I mass is about $7 \%$ of that of DDO 47 . The companion has been previously catalogued in the optical as CGCG 087-033. The geometry of the pair suggests that the relative orbits are within the plane of the sky. The projected distance $(20 \mathrm{kpc})$ is therefore likely a good approximation of the true seperation. 


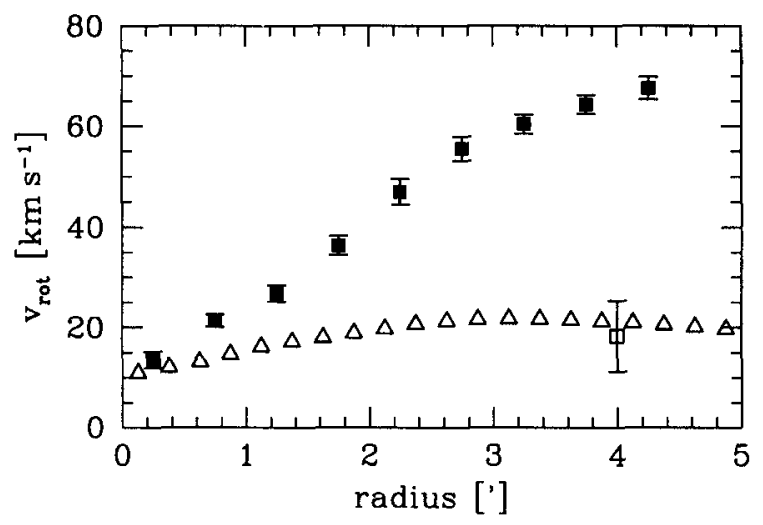

Figure 3. The measured rotation curve of DDO 47 (filled squares). For comparison, the contribtion from neutral hydrogen (triangles) and the stellar population (open square) is presented as well.

- To explain the derived rotation curve, DDO 47 must have a high Dark Matter content (about $80 \%$ of its dynamical mass).

- In total, $19 \mathrm{H} \mathrm{I}$ holes were detected, most of which are expanding. There is a striking correlation between the positions of the holes and current star forming regions (as traced by $\mathrm{H} \alpha$-observations) in the sense that current star formation is restricted to the rims of the shells.

- The thickness of the H I disk is about $700 \mathrm{pc}$. DDO 47 is therefore one of the thickest dwarf galaxies studied so far.

\section{References}

Kim, S., Staveley-Smith, L., Sault, R.J., Kesteven, M.J., McConnell, D., \& Freeman, K.C. 1997, PASA, 14, 119

Puche, D., Westpfahl, D., Brinks, E., \& Roy, J.-R. 1992, AJ, 103, 1841

Rhode, K.L., Salzer, J.J., \& Westpfahl, D.J. 1997, BAAS, 191, 81.09

Strobel, N.V., Hodge, P., Kennicutt, R.C. Jr. 1991, ApJ, 383, 148

Tenorio-Tagle, G., \& Bodenheimer, P. 1988, ARA\&A, 26, 145

Walter, F. \& Brinks, E. 1998, AJ, submitted 


\section{Searching for LSB - IV}

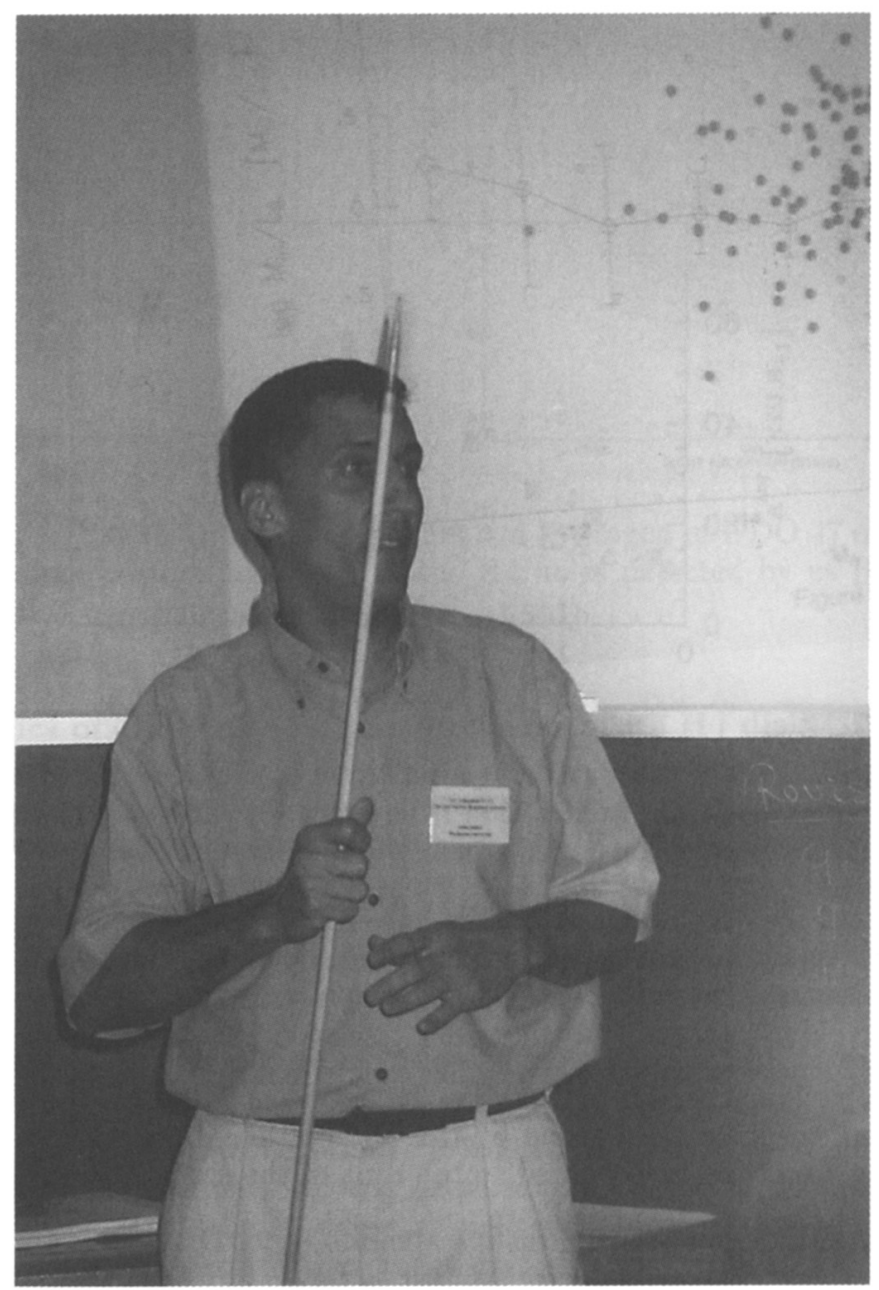

Call yourselves astronomers, it's up there - on the ceiling. 